\title{
Increased expression of Ero1L-alpha in healing fetal wounds
}

\author{
Phillip H Gallo, Latha Satish, Sandra Johnson and Sandeep Kathju*
}

\begin{abstract}
Background: Adult mammalian tissues heal injury to the skin with formation of scar; this process quickly seals an injured area, however, excessive scar formation can become a source of persistent pathology, interfering with multiple vital functions. In contrast, mammalian fetal tissue can heal without scar formation. We previously sought to model scarless healing in a rabbit fetal skin wound and identified gene products differentially expressed during fetal wound healing through PCR suppression subtractive hybridization (PCR SSH). One of these transcripts, previously identified simply as clone 11, showed putative increased expression in wounded fetal skin. This study establishes its identity as Ero1L-alpha and confirms its elevated expression in healing fetal wounds.
\end{abstract}

Findings: After obtaining further sequence by $5^{\prime}$ rapid amplification of cloned ends (RACE) we find that clone 11 is Ero1L-alpha. We determined that clone 11, a differentially expressed transcript in fetal wound healing, comprises the $3^{\prime}$ untranslated region (UTR) of an approximately $4 \mathrm{~kb}$ transcript in rabbit tissues that corresponds to Ero1Lalpha. We showed that Ero1L-alpha is expressed predominantly as two transcripts in rabbit skin, namely a $1.6 \mathrm{~kb}$ transcript and the $4.0 \mathrm{~kb}$ transcript recovered in our PCR SSH screen via its 3' UTR sequence. However, a third transcript of $2.9 \mathrm{~kb}$ was also detected in Northern blots and was subsequently cloned and confirmed by 3' RACE. Knockdown of the clone 11 sequence in rabbit adult fibroblasts via siRNA resulted in significantly decreased Ero1Lalpha message expression. Increased expression of clone 11 (Ero1L-alpha) in a variety of cell types during the wound healing response was also confirmed by in situ hybridization.

Conclusions: Ero1L-alpha is one of the previously unknown clones identified in a PCR SSH screen for genes differentially expressed in fetal wounded tissue. In situ hybridization confirms that Ero1L-alpha shows increased expression in multiple cell types after wounding of the fetal integument.

\section{Introduction}

Mammalian skin has multiple critical functions including providing homeostasis and serving as a first line of defence against infection. When injury to the skin occurs, a complex series of processes initiate its repair $[1,2]$. Adult (postnatal) mammals heal injury to the skin with attendant scar formation [3-5]; this process quickly seals an injured area, but excessive scar formation can become a source of persistent pathology and can interfere with numerous vital functions. In contrast, mammalian fetal skin can heal without scar formation. Much research has focused on identifying the mechanisms underlying scarless fetal wound repair; these experiments have primarily compared adult and fetal wound

\footnotetext{
* Correspondence: skathju@wpahs.org

Center for Genomic Sciences, Allegheny-Singer Research Institute, Allegheny General Hospital, Pittsburgh, PA 15212, USA
}

healing by examining various growth factors/cytokines, extracellular matrix proteins, and chaperonins [6-13]. To date, no specific critical pathways determinative for scarless repair have been established.

We have previously examined scarless wound healing in fetal skin by incisional wound modelling in rabbits [14]. Using PCR suppression subtraction hybridization (PCR SSH) we identified transcripts exhibiting differential expression during the fetal wound healing response. Because PCR SSH compares two conditions across the entire expressome and recovers only fragments of gene products, numerous genes of unknown identity and/or function were recovered. One of the unidentified gene products, designated clone 11, was upregulated in pooled samples of fetal wounded tissue samples 12 hours postwounding when compared to fetal unwounded control skin tissue. Herein, we present evidence identifying this 
transcript as Ero1L-alpha and confirming its elevated expression with in vivo studies.

\section{Materials and methods}

Source Tissues and Fibroblast Culture

All animal protocols were reviewed and approved by the Institutional Animal Care and Use Committee (IACUC); details of animal sample collection were as described in Kathju et al [14]. $\sim 1 \mathrm{~cm}$ incisional wounds were placed on the dorsums of fetal rabbits at 20-21 days gestation, then harvested 8 days later (as well as unwounded fetal control skin). For in situ hybridization studies, fetal wounded and unwounded control tissues were stored in 10\% Neutral Buffered Formalin (up to 7 days) before embedding; processing, paraffin embedding, and sectioning of samples were performed by Research Histology Services (Pittsburgh, PA) using standard conditions.

For fibroblast culture, samples of unwounded control skin were obtained from adult and fetal rabbits and were minced into small pieces within 30 minutes after dissection. The tissue samples were washed extensively in PBS containing $1 \mathrm{X}$ antibiotic/antimycotic solution (containing penicillin, streptomycin, and amphotericin B; Invitrogen Corporation, Carlsbad, CA) and then placed in RPMI 1640 medium (Invitrogen) containing
$10 \%$ fetal bovine serum (FBS, Gemini Bio-Products) and $1 \times$ antibiotic/antimycotic solution (Invitrogen). The cultures were left undisturbed for 7 days in a $37^{\circ} \mathrm{C}$ incubator containing $5 \% \mathrm{CO}_{2}$ supplement. The fibroblast outgrowths observed after a week from primary cultures were sub-cultured immediately using 0.5\% Trypsin EDTA (Invitrogen). Once the cells reached $90 \%$ confluence, they were either: 1) passaged once more before total RNA was isolated from passage three adult and fetal fibroblasts, or 2) plated onto 6-well plates, transfected with $100 \mu \mathrm{M}$ siRNA (sequences in Table 1 ) and 4 $\mu$ l Lipofectamine 2000 (Invitrogen) as previously described [15], and grown for 2 days before purification of total RNA.

\section{RNA extraction/purification of samples}

For all tissue culture RNA purifications, total RNA was obtained using the RNeasy Micro Kit (Qiagen Inc. USA, Valencia, CA) following manufacturer's protocols with a DNase treatment step. The quality of total RNA extracted from fetal and adult tissue and fibroblasts was examined by capillary electrophoresis using an Agilent 2100 Bio Analyzer (Agilent Technologies Inc., Palo Alto, CA), and the quantity determined using the $\mathrm{OD}_{260} / \mathrm{OD}_{280}$ ratio measured using a ND-1000 spectrophotometer (Nanodrop Technologies Inc., Wilmington, DE).

Table 1 Primers, probes, siRNAs used

\begin{tabular}{|c|c|c|}
\hline $\begin{array}{c}\text { Primer Number/Name } \\
\text { (from Figure } 2 A \text {, if applicable) }\end{array}$ & Direction & Primer Sequence \\
\hline 1 & $\mathrm{~F}$ & TCAGCCAGTGTGGAAGGAGGGA \\
\hline 2 & $\mathrm{~F}$ & AGGAGACGCAGAAGGCTGTTC \\
\hline 3 & $\mathrm{~F}$ & TTGGGTTGTTTTGGTGGTAGAAAGGT \\
\hline 4 & $\mathrm{~F}$ & GCCGACAGTCAGCAAGTTTGCTITATC \\
\hline 5 & $\mathrm{~F}$ & GCAGGGCTTGTGGATGTAATGTG \\
\hline 6 & $\mathrm{R}$ & CTGATGTCATTCCAGAAAGGAC \\
\hline 7 & $\mathrm{R}$ & TAACAGCACAGTCCCTCCTTCCACAC \\
\hline 8 & $\mathrm{R}$ & TCAGAGCAGTACCCAAACCCTG \\
\hline 9 & $\mathrm{R}$ & CGGAACAGCAATGGAGTTGGTAAG \\
\hline clone11 siRNA sense & & rGrUrGrCrUrArCrArCrArGrArCrCrUrGrUrCrUTT \\
\hline clone11 siRNA antisense & & rArGrArCrArGrGrUrCrUrGrUrGrUrArGrCrArCTT \\
\hline scrambled siRNA sense & & rUrGrCrGrArUrArCrGrArCrArUrCrCrUrCrGrUTT \\
\hline scrambled siRNA antisense & & rArGrUrArCrCrUrGrCrUrGrGrGrUrCrArGrArATT \\
\hline Ero1LA real time for & $\mathrm{F}$ & ACCTGAAGAGGCCTTGTCCTIT \\
\hline Ero1LA real time rev & $\mathrm{R}$ & TCCATCAGGAACTTCATCAGATTG \\
\hline Ero1LA real time probe & P & TGGAATGACATCAGCCAGTGTGGAAG \\
\hline clone11 real time for & $\mathrm{F}$ & CTCTGAAAACATGACTCCCTCCTT \\
\hline clone11 real time rev & $\mathrm{R}$ & AGGAGTCTGGCTTTCTCCTGAA \\
\hline clone11 real time probe & $P$ & CACCGCTCTGTGACCTCCTGAAC \\
\hline GAPDH real time for & $\mathrm{F}$ & СTCTGAAAACATGACTCCCTCCTT \\
\hline GAPDH real time rev & $\mathrm{R}$ & CCTCGGTGTAGCCCAGGAT \\
\hline GAPDH real time probe & $P$ & AAGCAGGCATCCGAGGGCCC \\
\hline
\end{tabular}




\section{RACE}

Rapid amplification of cloned ends (RACE) was used to obtain 3' UTR sequence for the various Ero1L-alpha clones. The GeneRacer kit with AMV reverse transcriptase (Invitrogen) was used for 3' RACE following manufacturer's directions; $2 \mu \mathrm{g}$ of total RNA from fetal control skin was used as the source RNA. All subsequent PCR was done using the AccuPrime HF PCR system (Invitrogen) and using the primers listed in Table 1 following manufacturer's directions. Cloned amplimers from RACE reactions were then sequenced.

Primers for Ero1L-alpha were designed from the predicted rabbit sequence for Ero1L-alpha (ENSOCUG00000012632) from Ensembl [16] and primers for GAPDH were designed from the NCBI rabbit GAPDH sequence (NM_001082253) [17].

\section{DIG RNA probes}

DNA constructs for DIG RNA probes were prepared by either DNA digests of plasmids bearing subcloned inserts or by direct PCR of cloned and sequenced probes; DNA templates were gel extracted before use. DIG RNA probes were prepared using the DIG RNA labelling kit (Roche, Indianapolis, IN) and T3 RNA Polymerase (Promega Corporation, Madison, WI). RNA was purified using the RNeasy Micro Kit (Qiagen) following manufacturer's protocols.

\section{Northern blot}

Northern blots were either commercially prepared (Zyagen, San Diego, CA) or prepared using passage three fetal and adult fibroblast RNA. Northern blots were prepared using reagents from the NorthernMax kit (Applied Biosystems/Ambion, Austin, TX) and with the NorthernMax Loading Dye without ethidium bromide. Total RNA was used as the source for preparing mRNA using the Oligotex kit (Qiagen). 100 nanograms of mRNA and RNA marker (Invitrogen) were separated on $1 \%$ denaturing formaldehyde gels and transferred to a positively charged nylon membrane (Roche). RNA was cross-fixed to the membrane using a UV light box (SpectroLinker XL-1000, optimal crosslink settings).

Blots were rinsed with DEPC-treated water, and then prehybridized in DIG EasyHyb buffer (Roche) following manufacturer's protocol. Blots were hybridized overnight at $68^{\circ} \mathrm{C}$ in DIG EasyHyb with 100 ng DIG-labeled probe. Blots were washed for two 5 minute low stringency washes in $2 \times \mathrm{SSC}, 0.1 \% \mathrm{SDS}$ at room temperature followed by two 20 minute high stringency washes in $0.2 \times \mathrm{SSC}, 0.1 \% \mathrm{SDS}$ at $68^{\circ} \mathrm{C}$. All blocking, washing and detection of DIG were done with the DIG wash and Block kit (Roche) and CDP-Star (Roche) following manufacturer's protocols.

\section{Quantitative real time RT-PCR}

The primer sets for rabbit clone 11 and rabbit Ero1Lalpha were designed using the initial sequence for clone 11 obtained by PCR SSH, plus additional sequence obtained through 5' RACE and 3' RACE, as well as the predicted rabbit sequence for Ero1L-alpha (ENSOCUG00000012632) from Ensembl [16]. The Taqman primers/probes reported in Table 1 were designed using Primer Express software (Applied Biosystems, Foster City, CA). Initial RT-PCR assays on $100 \mathrm{ng}$ of fetal unwounded control RNA were used to verify that each of the primer sets was detectable in fetal tissue and resulted in only a single amplicon of the expected molecular weight. Primer sequences for the rabbit GAPDH (used as an internal control) were previously published [14]. All primers and fluorocoupled Taqman probes were purchased from Integrated DNA Technologies (Coralville, IA).

$100 \mathrm{ng}$ of total RNA from samples was used for reverse transcriptase (RT) reaction (using gene-specific reverse primer and $10 \mu \mathrm{l}$ of total volume); for subsequent real time PCR assays, $1.5 \mu \mathrm{L}$ of $\mathrm{RT}$ reaction, 800 $\mathrm{nM}$ of each primer, and $160 \mathrm{nM}$ of the appropriate probe (final concentrations) in a total volume of $15 \mu \mathrm{l}$ were used. The remaining protocol parameters for RT reaction and real time PCR were followed as previously described [14]. Using the comparative critical cycle (Ct) method and using GAPDH as the endogenous control, the expression levels of the target genes were normalized and the relative abundance was calculated. Results shown are representative of three independent experiments performed in triplicate; statistical analysis for significance was performed using a Student's t-test.

\section{in situ Hybridizations}

Sectioned slides of unwounded fetal skin or wounded fetal skin at 8 days post-injury were processed as described [18], except using paraffin embedded sections. Slides were deparaffinated using three xylene washes and passed through an ethanol series, with a final wash in $1 \times$ PBS. Slides were then processed as described [18], using a 48 hour hybridization at $58^{\circ} \mathrm{C}$. Slides were counterstained with $0.1 \%$ Nuclear FastRed (Vector Labs, Burlingame, CA) and mounted with aqueous ImmuMount (ThermoFisher, Pittsburgh, PA) before photographing.

\section{Results/Discussion}

Our original PCR SSH screen [14] recovered multiple fragments of gene sequence that are putatively over- or under-expressed in healing fetal wounds compared to unwounded fetal control tissues, but to which an exact identity could not be assigned. To better characterize these gene fragments, we interrogated a commercially available rabbit multi-tissue Northern blot with 
non-radioactive DIG probes directed against clones identified in our screen. When the Northern blot was hybridized with a probe directed against one gene product from our screen, previously identified simply as clone 11, a single major transcript of approximately 4 $\mathrm{kb}$ was detected in all rabbit adult tissues (Figure 1), with highest levels of expression in the lungs, kidneys, heart, ovaries, and testis, and lower levels of expression in the pancreas.

We originally obtained only several hundred base pairs of clone 11 sequence from our PCR SSH screen. We then undertook both 5' and 3' RACE to obtain further contiguous clone 11 sequence in the hopes of establishing its identity. Using the Ensembl rabbit database and BLAST, we determined that this expanded clone 11 sequence was derived from a rabbit genomic region approximately $2.5 \mathrm{~kb}$ from the predicted gene Ero1Lalpha. In humans, two transcripts of Ero1L-alpha are known to exist; both contain a 1290 bp coding region but differ in the length of their 3' UTRs, with the longer one approximating $5.3 \mathrm{~kb}$ in total length.

We therefore hypothesized that clone 11 in the rabbit was the extended 3' UTR form of Ero1L-alpha. Using the rabbit predicted Ero1L-apha sequence from Ensembl (ENSOCUG00000012632), we designed primers to amplify (by RT-PCR) a large portion of the Ero1L-alpha coding region from rabbit fetal control RNA. This amplimer was subcloned and sequenced and matched the predicted rabbit sequence from Ensembl, with 92\% sequence similarity to human Ero1L-alpha and 97\% predicted protein similarity within the coding region of Ero1L-alpha.

To confirm that the clone 11 sequence obtained from our screen was the transcript of Ero1L-alpha containing an extended 3' UTR, we designed siRNA against the clone 11 sequence and transfected rabbit adult fibroblasts with clone 11-specific siRNA, scrambled control

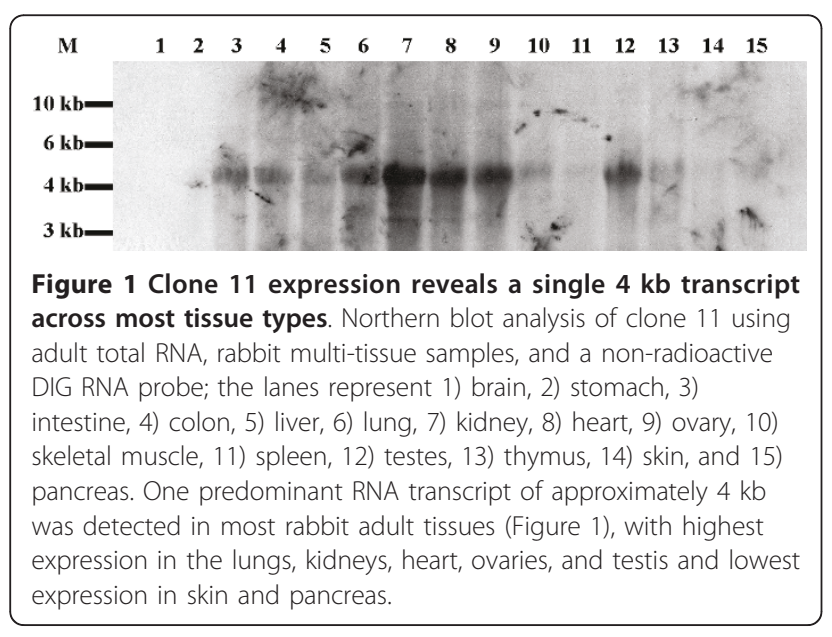

siRNA, or mock-transfected. After 48 hours, the RNA purified from the various transfected fibroblast populations was reverse transcribed using a gene specific primer located within clone 11 sequence, and subjected to real time PCR with probes specific either to clone 11 or to the Ero1L-alpha coding region (Figure 2C). Due to the use of clone 11 sequence as the primer for reverse transcription, located in the extended 3' UTR of the presumed Ero1L-alpha transcript isoform, the real time PCR probe designed against the coding sequence of Ero1L-alpha could only detect the extended 3' UTR transcript. We found that the siRNA against clone 11 significantly reduced detection of both clone 11-specific and Ero1L-alpha coding sequence-specific real time PCR probes. Neither scrambled siRNA nor mock transfection resulted in any Ero1L-alpha message decrease, confirming that our clone 11 sequence is the 3' terminus of the extended $4 \mathrm{~kb}$ Ero1L-alpha transcript. As a further confirmation of these results, mouse NIH $3 \mathrm{~T} 3$ cells were transfected with siRNA against sequence corresponding to clone 11, and demonstrated a similar reduction in Ero1L-alpha coding transcript (data not shown).

Using a DIG-labelled probe derived from rabbit Ero1L-alpha coding sequence, we sought to determine which transcript isoforms are expressed in fetal and adult skin tissues and fibroblasts, and to confirm that Ero1L-alpha is expressed as two major transcripts in the rabbit as was previously reported in humans. Using a Northern blot prepared with total RNA isolated from fetal and adult unwounded skin tissues and from fetal and adult fibroblasts in culture, two transcripts of the expected molecular weights ( 1400 bp and $\sim 4 \mathrm{~kb})$ were in fact observed in all samples (Figure $2 \mathrm{~B}$ ). Interestingly and surprisingly, a third minor message isoform corresponding to some $2.9 \mathrm{~kb}$ in length was also detected. We have subsequently used 3' RACE (using primers within the Ero1L-alpha coding sequence) to amplify and subclone the 3' end of this isoform, and confirm that it carries coding sequence identical to the other two transcripts, but with an intermediate length 3' UTR (data not shown). A full schematic, showing the various Ero1L-alpha message isoforms, together with locations of probes and primers is depicted in Figure 2A.

Finally, we sought to determine if the long $(4 \mathrm{~kb})$ variant of Ero1L-alpha is indeed increased after fetal wounding, as would be expected from its survival in the PCR SSH screen, and in which cell types this might be evident. To address this question, fetal control and wounded tissues were paraffin embedded, sectioned onto slides, and used for in situ hybridization (Figure 3). DIG-labeled RNA probes against the original clone 11 sequence were used to determine that healing fetal wound tissue displayed a greater level of expression as compared to control tissue from unwounded littermates 

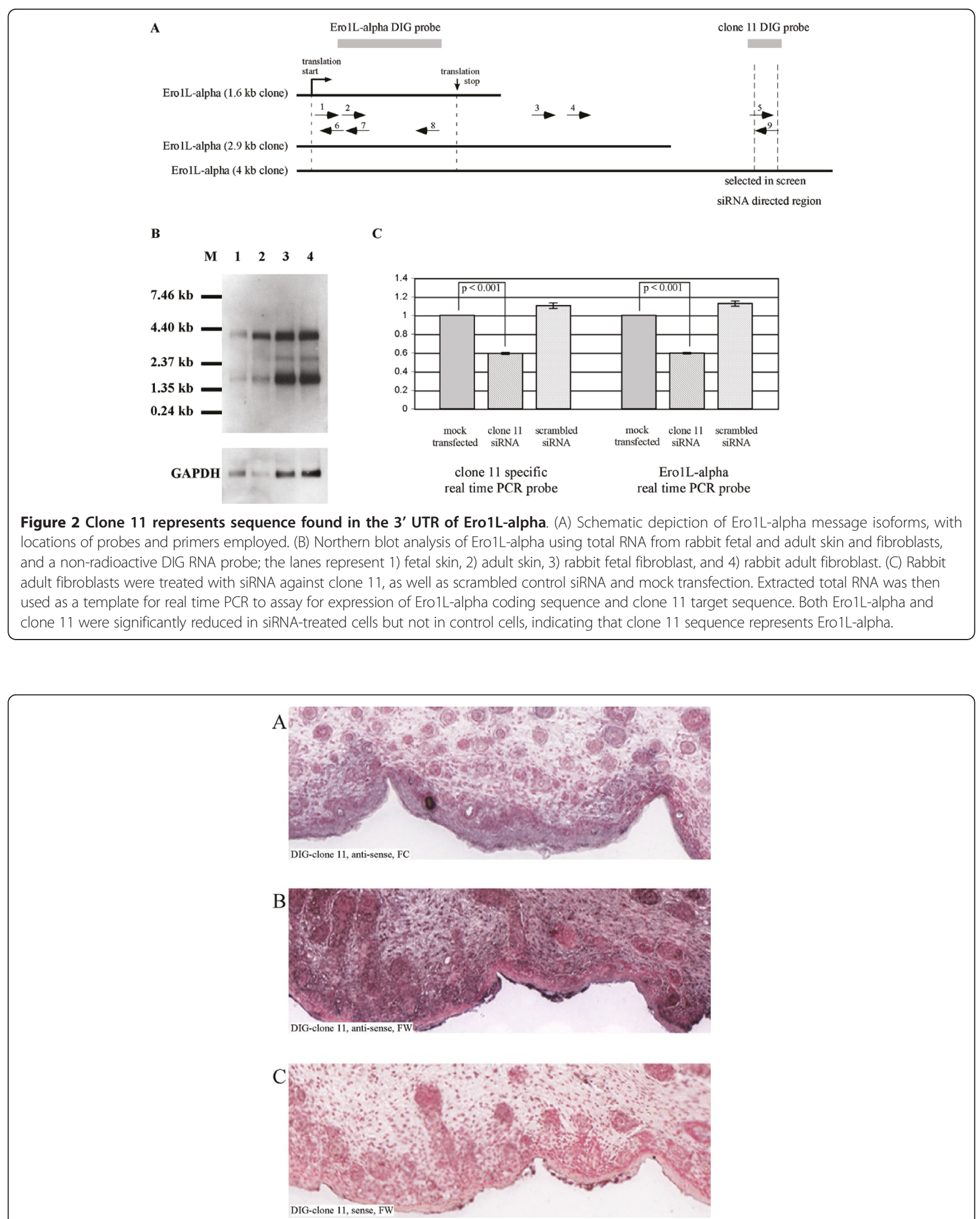

Figure 3 Increased expression of Ero1L-alpha in healing fetal wound. In situ hybridization using probe against clone 11 sequence reveals that Ero1L-alpha is over-expressed in multiple cell types after fetal skin wounding (3B) compared to unwounded control skin (3A). (3C): healing fetal wound tissue probed with a sense probe to clone 11 , which would not be expected to hybridize to the target sequence. All images are at $5 \times$ magnification. 
(Figure 3). A substantially higher intensity of staining was observed when antisense probe was used on wounded fetal tissue (Figure 3B) compared to unwounded fetal control tissue (Figure 3A) Minimal staining was observed in wounded tissue using sense probe (Figure $3 \mathrm{C}$ ) attesting that the assay is detecting the correct RNA target. Interestingly, the $4 \mathrm{~kb}$ Ero1Lalpha isoform appears to show increased expression in almost all cell types within or adjacent to the zone of healing injury. To ensure that our observed over-expression of Ero1L-alpha was not due to differences in RNA levels due to sample preparation, a rabbit probe against GAPDH was generated and tested in in situ hybridization as well. This probe demonstrated no major difference in staining intensity between the fetal control and wounded samples (data not shown), confirming that the increased expression seen in Ero1L-alpha is a specific event.

Ero1L-alpha plays a major role in the oxidative protein folding pathway in the endoplasmic reticulum, enabling proteins to form disulfide bonds [19], and is specifically up-regulated in response to hypoxic conditions [20]. It has not been previously implicated in wound healing, but it is not surprising that its expression may be elevated in response to some hypoxic or ischemic stimulus at the zone of injury. It is interesting that we recovered only the $4 \mathrm{~kb}$ variant of Ero1L-alpha transcript in our PCR SSH screen, with its attendant lengthy 3' UTR. Such UTR sequences have been found in other systems to regulate gene expression through post-transcriptional mechanisms [21-24], and it may be that a similar phenomenon is occurring here. More study will be required to fully explicate the specific functional and regulatory significances of the multiple Ero1L-alpha isoforms we have identified.

\section{Conclusion}

We conclude that one of the previously unidentified gene fragments recovered in a PCR SSH screen examining fetal wound healing, clone 11 , is a $4 \mathrm{~kb}$ isoform of Ero1L-alpha, featuring an extended 3' UTR sequence. Ero1L-alpha is expressed as three distinct mRNA isoforms in fetal and adult skin tissues and fibroblasts, including a novel $2.9 \mathrm{~kb}$ variant. The $4 \mathrm{~kb}$ isoform of Ero1L-alpha shows increased expression in multiple cell types after fetal integumentary wounding by in situ hybridization.

\footnotetext{
Acknowledgements

This study was supported by the Allegheny Singer Research Institute, Allegheny General Hospital and Pittsburgh Tissue Engineering Institute (PTEI). This work was funded from the grants awarded to S.K. (DE 014780), and L.S. (3M Fellowship). We thank Dr. Melissa Gallo for helpful discussions and critical reading of the manuscript. We extend our thanks to Ms. Mary O'Toole for her assistance in preparing this manuscript.
}

\section{Authors' contributions}

PHG, LS and SK designed the study. PHG performed the majority of the experimental work. SJ assisted with animal surgery, sample collection and RNA purification. LS derived fibroblast cultures and performed initial siRNA experiments. PHG, SK, and LS drafted the manuscript. All authors critically reviewed the final manuscript

\section{Competing interests}

The authors declare that they have no competing interests.

Received: 15 November 2010 Accepted: 6 June 2011

Published: 6 June 2011

\section{References}

1. DePalma RL, Krummel TM, Durham LA III, Michna BA, Thomas BL, Nelson JM, Diegelmann RF: Characterization and quantitation of wound matrix in the fetal rabbit. Matrix 1989, 9:224-31.

2. Martin P: Wound healing-aiming for perfect skin regeneration. Science 1997, 276:75-81.

3. Clark RAF: The Molecular and Cellular Biology of Wound Repair. New York: Plenum Press; 21995.

4. Rowlatt U: Intrauterine wound healing in a 20 week human fetus. Virchows Arch A Pathol Anat Histol 1979, 381:353-61.

5. Armstrong $J R$, Ferguson MWJ: Ontogeny of the skin and transition from scar free to scarring phenotype during wound healing in the pouch young of Monodelphis domestica. Dev Biol 1995, 169:242-60.

6. Colwell AS, Longaker MT, Lorenz HP: Fetal wound healing. Front Biosci 2003, 8:51240-8.

7. Lorenz HP, Longaker MT, Perkocha LA, Jennings RW, Harrison MR, Adzick NS: Scarless wound repair: a human fetal skin model. Development 1992, 114:253-9.

8. Bullard KM, Longaker MT, Lorenz HP: Fetal wound healing: current biology. World J Surg 2003, 27:54-61.

9. Houghton PE, Keefer KA, Krummel TM: The role of transforming growth factor beta in the conversion from scarless healing to healing with scar formation. Wound Repair Regen 1995, 229-36.

10. Haynes JH, Johnson DE, Mast BA, Diegelmann RF, Salzberg DA, Cohen IK Krummel TM: Platelet-derived growth factor induces fetal wound fibrosis. J Pediatr Surg 1994, 2:1405-8.

11. Liechty KW, Kim HB, Adzick NS, Crombleholme TM: Fetal wound repair results in scar formation in interleukin-10 deficient mice in a syngeneic murine model of scarless fetal wound repair. J Pediatr Surg 2000, 35:866-72

12. Soo C, Hu FY, Zhang X, Wang Y, Beanes SR, Lorenz HP, Hedrick MH, Mackool RJ, Plaas A, Kim SJ, Longaker MT, Freymiller E, Ting K: Differential expression of fibromodulin, a transforming growth factor beta modulator, in fetal skin development and scarless repair. Am J Path 2000, 157:423-33.

13. Darden DL, Hu FZ, Ehrlich MD, Gorry MC, Dressman D, Li HS, Whitcomb DC, Hebda PA, Dohar JE, Ehrlich GD: RNA differential display of scarless wound healing in fetal rabbit indicates downregulation of a CCT chaperonin subunit and upregulation of a glycophorin-like gene transcript. J Pediatr Surg 2000, 35:406-19.

14. Kathju S, Satish L, Rabik C, Rupert T, Oswald D, Johnson S, Hu FZ, Ehrlich GD: Identification of differentially expressed genes in scarless wound healing utilizing polymerase chain reaction-suppression subtraction hybridization. Wound Repair Regen 2006, 14:413-20.

15. Satish L, Johnson S, Wang JH, Post JC, Ehrlich GD, Kathju S: Chaperonin containing T-complex polypeptide subunit eta (CCT-eta) is a specific regulator of fibroblast motility and contractility. PLoS One 2010, 5(4): e10063.

16. Ensembl. [http://www.ensembl.org].

17. NCBI. [http://www.ncbi.nlm.nih.gov]

18. Braissant O, Wahli W: A simplified in situ hybridization protocol using non-radioactively labelled probes to detect abundant and rare mRNAs on tissue sections. Biochemica 1998, 1:10-16.

19. Gess B, Hofbauer K, Wenger R, Lohaus C, Meyer H, Kurtz A: The cellular oxygen tension regulates expression of the endoplasmic oxidoreductase Ero1L-alpha. Eur J Biochem 2003, 270:2228-2235.

20. Tu BP, Weissman JS: Oxidative protein folding in eukaryotes: mechanisms and consequences. J Cell Biol 2004, 164:341-346. 
21. Hughes TA: Regulation of gene expression by alternative untranslated regions. Trends Genet 2006, 22(3):119-122.

22. Lutz CS: Alternative polyadenylation: a twist on mRNA 3 ' end formation. ACS Chem Biol 2008, 3(10):609-617.

23. Edwalds-Gilbert G, Veraldi KL, Milcarek C: Alternative poly(A) site selection in complex transcription units: means to an end? Nuc Acids Res 1997, 25(13):2547-2561.

24. Danckwardt S, Hentze MW, Kulozik AE: $3^{\prime}$ end mRNA processing:

molecular mechanisms and implications for health and disease. EMBO $J$ 2008, 27:482-498.

doi:10.1186/1756-0500-4-175

Cite this article as: Gallo et al:: Increased expression of Ero1L-alpha in healing fetal wounds. BMC Research Notes 2011 4:175.

Submit your next manuscript to BioMed Central and take full advantage of:

- Convenient online submission

- Thorough peer review

- No space constraints or color figure charges

- Immediate publication on acceptance

- Inclusion in PubMed, CAS, Scopus and Google Scholar

- Research which is freely available for redistribution

Submit your manuscript at www.biomedcentral.com/submit 